\title{
Regional Differences in Efficacy, Safety, and Biomarkers for Second-Line Axitinib in Patients with Advanced Hepatocellular Carcinoma: From a Randomized Phase II Study
}

\author{
Masatoshi Kudo ${ }^{a}$ Yoon-Koo Kang ${ }^{b}$ Joong-Won Park ${ }^{c}$ Shukui Qin ${ }^{d}$ \\ Yoshitaka Inaba ${ }^{\mathrm{e}}$ Eric Assenat ${ }^{\mathrm{f}}$ Yoshiko Umeyamag Maria José Lechuga ${ }^{\mathrm{h}}$ \\ Olga Valotah $^{\text {h }}$ Yosuke Fujii ${ }^{g}$ Jean-Francois Martini ${ }^{i} \quad$ J. Andrew Williams ${ }^{i}$ \\ Shuntaro Obij \\ ${ }^{a}$ Department of Gastroenterology and Hepatology, Kindai University Faculty of Medicine, \\ Osaka-Sayama, Japan; ${ }^{b}$ Department of Oncology, Asan Medical Center, University of Ulsan \\ College of Medicine, Seoul, and ' Center for Liver Cancer, National Cancer Center, Goyang, \\ Republic of Korea; d Department of Medical Oncology, Nanjing Bayi Hospital, Nanjing, China; \\ e Department of Diagnostic and Interventional Radiology, Aichi Cancer Center Hospital,

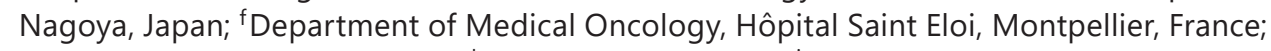

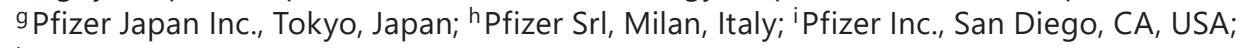 \\ jDepartment of Hepatology, Sasaki Foundation Kyoundo Hospital, Tokyo, Japan
}

\section{Keywords}

Asian $\cdot$ Axitinib $\cdot$ Biomarkers $\cdot$ Hepatocellular carcinoma $\cdot$ MicroRNAs

\begin{abstract}
Background: An unmet need exists for treatment of patients with advanced hepatocellular carcinoma (HCC) who progress on or are intolerant to sorafenib. A global randomized phase II trial (ClinicalTrial.gov No. NCT01210495) of axitinib, a vascular endothelial growth factor receptor 1-3 inhibitor, in combination with best supportive care (BSC) did not prolong overall survival (OS) over placebo/BSC, but showed improved progression-free survival in some patients. Subgroup analyses were conducted to identify potential predictive/prognostic factors. Methods: The data from this phase II study were analyzed for the efficacy and safety of axitinib/BSC in patients from Asia versus non-Asia versus Asian subgroups (Japan, Korea, or mainland China/Hong Kong/Taiwan) and predictive/prognostic values of baseline microRNAs
\end{abstract}

M.K. and Y.-K.K. equally contributed to the study and share first authorship. J.A.W. was employed at Pfizer at the time of the study. 


\section{Liver

Kudo et al.: Regional Differences in Efficacy, Safety, and Biomarkers for Second-Line

Axitinib in Patients with Advanced Hepatocellular Carcinoma

and serum soluble proteins, using the Cox proportional hazards model. Results: Of 202 patients, 78 were from non-Asia and 124 from Asia (37 Japanese, 36 Korean, and 51 Chinese). No significant differences in OS were found between axitinib/BSC and placebo/BSC in nonAsians, Asians, or Asian subgroups. However, in an exploratory analysis, axitinib/BSC showed favorable OS in Asians, especially Japanese, when patients intolerant to prior antiangiogenic therapy were excluded from the data set. Axitinib/BSC was well tolerated by non-Asians and Asians alike. The presence of 4 circulating microRNAs, including miR-5684 and miR-1224-5p, or a level lower than or equal to the median protein level of stromal cell-derived factor 1 at baseline was significantly associated with longer OS in axitinib/BSC-treated Asians or nonAsians. Conclusions: Axitinib/BSC did not prolong survival over placebo/BSC in non-Asians, Asians, or Asian subgroups, but favorable OS with axitinib/BSC was observed in a subset of Japanese patients. A patient population that excludes sorafenib-intolerant patients might potentially be more suitable for clinical trials of new agents in advanced HCC. Since these results are very preliminary, further investigation is warranted. The potential predictive/prognostic value of several baseline microRNAs and soluble proteins identified in this study would require validation in prospective studies on a large cohort of patients.

(C) 2017 S. Karger AG, Basel

\section{Introduction}

The incidence of liver cancer varies geographically, with higher incidence rates observed in developing countries in Asia and Africa than in developed countries in Europe and North America [1]. The etiology of hepatocellular carcinoma (HCC), which accounts for the majority of liver cancers, is diverse and complex, with hepatitis B virus being the primary cause of HCC, particularly in Asia (other than Japan) and Africa. For resectable HCC, treatment options include resection, locoregional therapy, ablation, or external-beam radiation therapy, whereas for unresectable, advanced, or metastatic HCC, systemic therapy with sorafenib is one of few treatments recommended by the National Comprehensive Cancer Network [2]. Sorafenib is so far the only targeted agent approved for advanced HCC, but its efficacy is limited and shortlived, which necessitates the evaluation of novel agents and/or new treatment approaches in this setting and later lines of therapy.

Several agents targeting the vascular endothelial growth factor (VEGF)/VEGF receptor (VEGFR) signaling pathway, including the multitargeted tyrosine kinase inhibitor brivanib [3] and the monoclonal antibody ramucirumab [4], or the mechanistic target of rapamycin inhibitor everolimus [5] were investigated in 2009-2013 in patients with sorafenibrefractory and -intolerant HCC in randomized phase III trials with generally disappointing results.

Axitinib is a potent and selective inhibitor of VEGFRs 1-3, approved for treatment of second-line metastatic renal cell carcinoma (RCC) [6]. Based on the antitumor activity of agents inhibiting the VEGF/VEGFR signaling pathway observed in HCC in clinical studies and the nonclinical activity of axitinib in HCC animal models, the efficacy and safety of axitinib in combination with best supportive care (BSC) was compared with placebo plus BSC (placebo/ BSC) in patients with locally advanced or metastatic HCC following failure of one prior antiangiogenic therapy in a global randomized phase II study conducted in 2010-2012 [7]. Although the study did not meet its primary endpoint of overall survival (OS), improvements favoring the axitinib/BSC arm were observed in progression-free survival (PFS), time to tumor progression, and the clinical benefit rate, especially among Asian patients. Furthermore, an exploratory analysis indicated a longer OS with axitinib/BSC than with placebo/BSC when patients who were intolerant to prior antiangiogenic therapy were excluded from the data set 


\section{Liver Cancer}

\begin{tabular}{l|l}
\hline Liver Cancer 2018;7:148-164 \\
\hline DOI: 10.1159/000484620 & $\begin{array}{l}\text { ○ 2017 S. Karger AG, Basel } \\
\text { www.karger.com/lic }\end{array}$ \\
\hline
\end{tabular}

Kudo et al.: Regional Differences in Efficacy, Safety, and Biomarkers for Second-Line Axitinib in Patients with Advanced Hepatocellular Carcinoma

[7]. The safety profile in patients with metastatic HCC was as expected based on prior studies of axitinib in RCC, with no new safety signals or unexpected toxicities.

The aims of the current analyses were to explore the efficacy and safety of axitinib/BSC compared with placebo/BSC in patients from non-Asia versus Asia versus Asian subgroups by country of origin (Japan; Korea; and mainland China, Hong Kong, and Taiwan combined [CHT]), and to investigate the potential predictive and/or prognostic value of baseline levels of circulating microRNAs (miRNAs) and serum soluble proteins for OS in the overall population as well as in non-Asian and Asian patients.

\section{Subjects and Methods}

\section{Study Design and Patients}

The data from the global randomized double-blind phase II trial (ClinicalTrials.gov No. NCT01210495) of axitinib/BSC versus placebo/BSC in patients previously treated with antiangiogenic therapy for locally advanced or metastatic HCC were analyzed in this study. The details of the study design and patient eligibility criteria have been reported previously [7]. In brief, patients with confirmed HCC who progressed on or were intolerant to one prior antiangiogenic therapy, with Child-Pugh class A liver function, and with an Eastern Cooperative Oncology Group performance status (ECOG PS) of 0 or 1 were stratified by tumor invasion (presence vs. absence of extrahepatic spread and/or vascular invasion) and geographic region (non-Asia vs. Asia) and randomly assigned in a 2:1 ratio to axitinib/BSC or placebo/BSC. The primary endpoint was OS, and secondary endpoints included PFS, safety, and assessment of baseline levels of circulating miRNAs and serum soluble proteins for their predictive and/or prognostic potential.

\section{Treatment and Assessments}

As described previously [7], the randomized patients received axitinib or placebo orally at a starting dose of $5 \mathrm{mg}$ twice daily in 4-week cycles. The axitinib or placebo dose could be increased to $7 \mathrm{mg}$ twice daily, and then to a maximum of $10 \mathrm{mg}$ twice daily, if patients tolerated the drug without any treatment-related adverse events (AEs) of grade $>2$ for 2 consecutive weeks and were normotensive. The drug dose could be decreased to $3 \mathrm{mg}$ twice daily, and then to $2 \mathrm{mg}$ twice daily, to manage axitinib-related AEs.

Radiological tumor assessments were performed by the investigators at baseline and every 8 weeks according to the Response Evaluation Criteria in Solid Tumors (RECIST) version 1.1. Safety was monitored throughout the study period, and AEs were graded per the National Cancer Institute Common Terminology Criteria for Adverse Events (NCI-CTCAE) version 3.0.

For evaluation of circulating miRNAs or serum soluble proteins, 5- or 10-mL whole-blood samples were collected from all randomized patients before administration of axitinib or placebo on cycle 1 day 1 and at the end of the study, and 2-3 h after dose administration on cycle 2 day 1 . After extraction with Agilent $8 \times$ 60K miRNA arrays (Release 19.0; www.genomics.agilent.com), human miRNAs were assessed using probes based on the latest Sanger miRBase (release 12.0; www.mirbase.org) at ShanghaiBio Corporation, Shanghai, China. The miRNA array data were extracted and processed using Agilent's proprietary Feature Extraction Software version 10.7.1.1 and were further normalized via the quantile method using Bioconductor $\mathrm{R}$ software. A total of 2,006 probes were measured from 170 patients. However, $78.8 \%$ of these miRNAs were not expressed at detectable levels; hence, the analyses correlating circulating miRNAs with efficacy were restricted to those miRNAs that were detected in at least 10 patients. Serum soluble proteins tested included angiopoietin (Ang)-2, matrix metalloproteinase 2, VEGF-A, VEGF-C, soluble VEGFR (sVEGFR)2, sVEGFR3, hepatocyte growth factor, soluble c-MET, stem cell factor (the ligand for c-KIT), neutrophil gelatinase-associated lipocalin, stromal cell-derived factor 1 (SDF-1), interleukin (IL)-6, IL-8, E-selectin, macrophage migration inhibitory factor (MIF), and chemokine (C-C motif) ligand 5 (also known as RANTES). Serum concentrations of proteins were assessed by Aushon BioSystems (Billerica, MA, USA) using the validated Aushon SearchLight Multiplex Analysis platform or an enzyme-linked immunosorbent assay.

\section{Statistical Analysis}

In the subgroup and exploratory analyses of the regional differences, OS and PFS was estimated using the Kaplan-Meier method, and the median and 95\% confidence interval (CI) were provided. An unstratified one-sided log-rank test was used to compare OS and PFS between the treatment arms, using a 0.025 signifi- 


\section{Liver Cancer}

\begin{tabular}{l|l}
\hline Liver Cancer 2018;7:148-164 \\
\hline DOI: 10.1159/000484620 & $\begin{array}{l}\text { @ 2017 S. Karger AG, Basel } \\
\text { www.karger.com/lic }\end{array}$ \\
\hline
\end{tabular}

Kudo et al.: Regional Differences in Efficacy, Safety, and Biomarkers for Second-Line Axitinib in Patients with Advanced Hepatocellular Carcinoma

cance level for interpretation. The hazard ratio (HR) and 95\% CI were also estimated. For miRNA predictive and/or prognostic factor analysis, a Cox proportional hazards model was used to estimate the HR and 95\% CI. A predictive effect was assessed as the interaction effect in this model, including main effects, and the interaction of treatment stratum with baseline miRNA expression status. A prognostic effect was assessed as the covariate effect in this model, including miRNA expression status stratified by treatment. The $p$ value was calculated using the Wald test. In the subgroup analyses of baseline level of soluble proteins, an unstratified two-sided log-rank test was used to compare OS between the treatment arms. In the exploratory analyses of miRNAs and soluble proteins, the Benjamini-Hochberg procedure was used with the false discovery rate (FDR) at the level of $0.2[8]$.

\section{Results}

\section{Patients}

In this multinational study, 78 patients were enrolled from non-Asian countries, whereas the majority $(n=124)$ were from Asian countries (Japan [ $n=37]$, Korea [ $n=36]$, and CHT [ $n=$ 51]). Of a total of 202 randomized patients, 134 ( 51 non-Asians and 83 Asians) were assigned to axitinib/BSC and 68 (27 non-Asians and 41 Asians) to placebo/BSC. The median age was not significantly different between non-Asian and all Asian patients (65.0 vs. 61.0 years). However, the patients in Japan were older (median 74.0 years), whereas the patients in Korea and CHT were younger (median 57.0 and 55.0 years, respectively), than those in non-Asia. The patient demographics and baseline characteristics of the axitinib/BSC and placebo/BSC arms of the non-Asian and Asian groups, as well as of the Asian subgroups, are summarized in Table 1. The percentage of female patients was slightly imbalanced between the axitinib/ BSC and placebo/BSC arms, except in CHT ( 9 and 11\%, respectively). It was higher in the axitinib/BSC arm than in the placebo/BSC arm in non-Asia (31 vs. 11\%), but it was lower in Asia (10 vs. $22 \%$ ), Japan (15 vs. $36 \%$ ), and Korea (4 vs. $27 \%$ ).

The baseline clinical characteristics, such as ECOG PS, the presence of tumor vascular invasion, or liver cirrhosis, were generally comparable between the two treatment arms, as well as between the non-Asian group, the Asian group, and the Asian subgroups. The majority of the patients in Japan had an ECOG PS of 0. All patients had Child-Pugh class A liver function. However, the percentage of patients with extrahepatic spread or hepatitis B, although similar between the treatment arms in each geographical region, was substantially higher in Asia, particularly in Korea and CHT, than in non-Asia. Based on the Barcelona Clinic Liver Cancer (BCLC) stage classification, a higher percentage of the Asian than of the non-Asian patients had a poorer prognosis of stage $C$, whereas the percentage of patients with BCLC stage $B$ was higher in non-Asia than in Asia. Of note, the percentage of patients with hepatitis $\mathrm{C}$ was highest in Japan compared with Korea, CHT, or non-Asia.

Prior locoregional therapy had been administered more frequently in Asia, especially in Japan and Korea, than in non-Asia. The majority of patients in both the axitinib/BSC and the placebo/BSC arm in each geographical region had previously received systemic therapy with sorafenib. However, the percentage of patients who were intolerant to prior sorafenib treatment (i.e., patients who discontinued prior antiangiogenic therapy due to treatmentrelated grade 3/4 AEs per NCI-CTCAE version 3.0) was higher in the placebo/BSC arm than in the axitinib/BSC arm in each region except in CHT (Table 1).

\section{Patient Disposition and Drug Exposure}

At the time of data cutoff (March 3, 2014), a similar number of patients in the axitinib/ BSC and the placebo/BSC arm in the non-Asian and Asian groups and the Asian subgroups discontinued the study, with death as the primary reason; almost all patients discontinued 
Kudo et al.: Regional Differences in Efficacy, Safety, and Biomarkers for Second-Line Axitinib in Patients with Advanced Hepatocellular Carcinoma

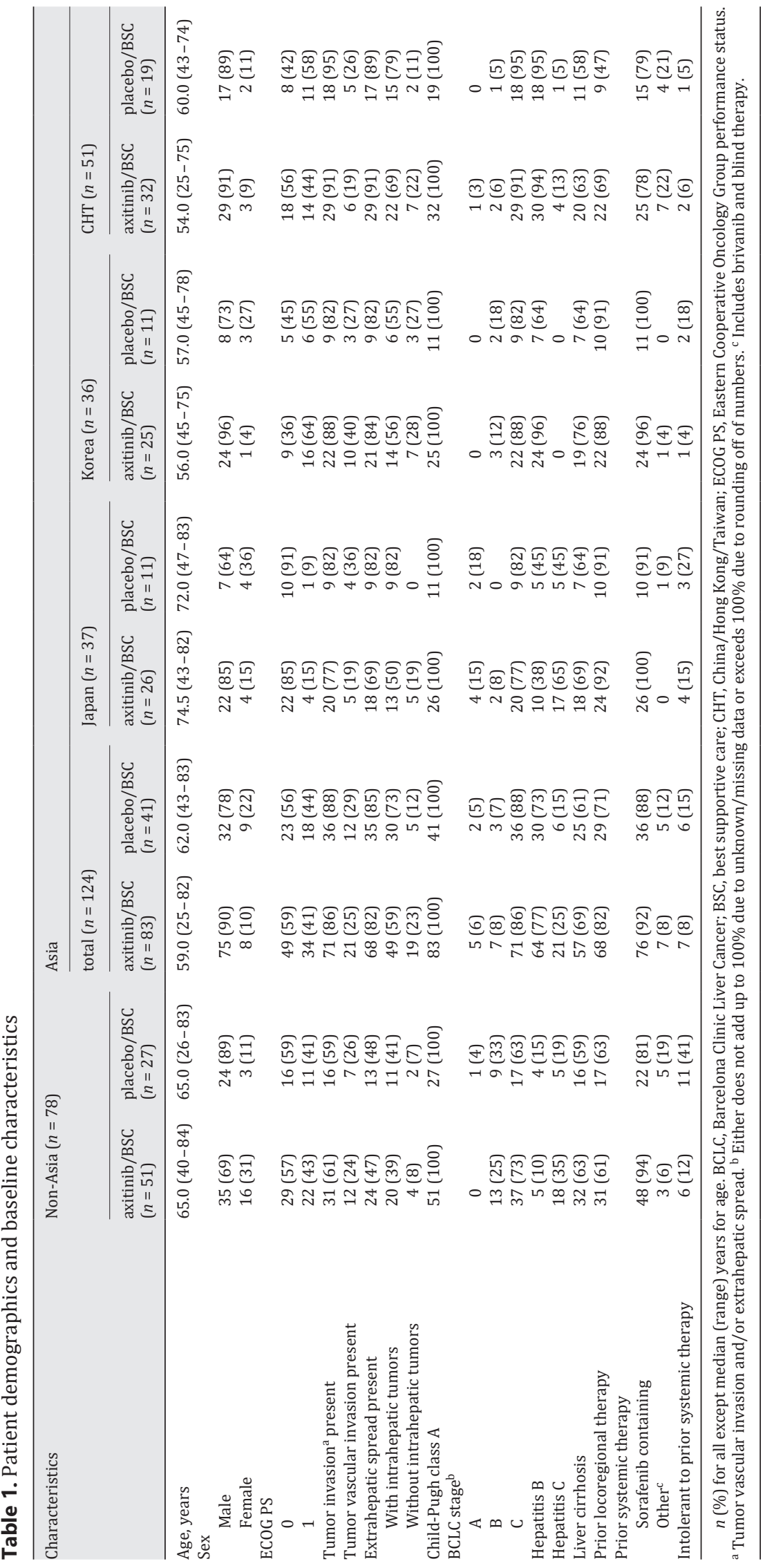


Kudo et al.: Regional Differences in Efficacy, Safety, and Biomarkers for Second-Lin Axitinib in Patients with Advanced Hepatocellular Carcinoma
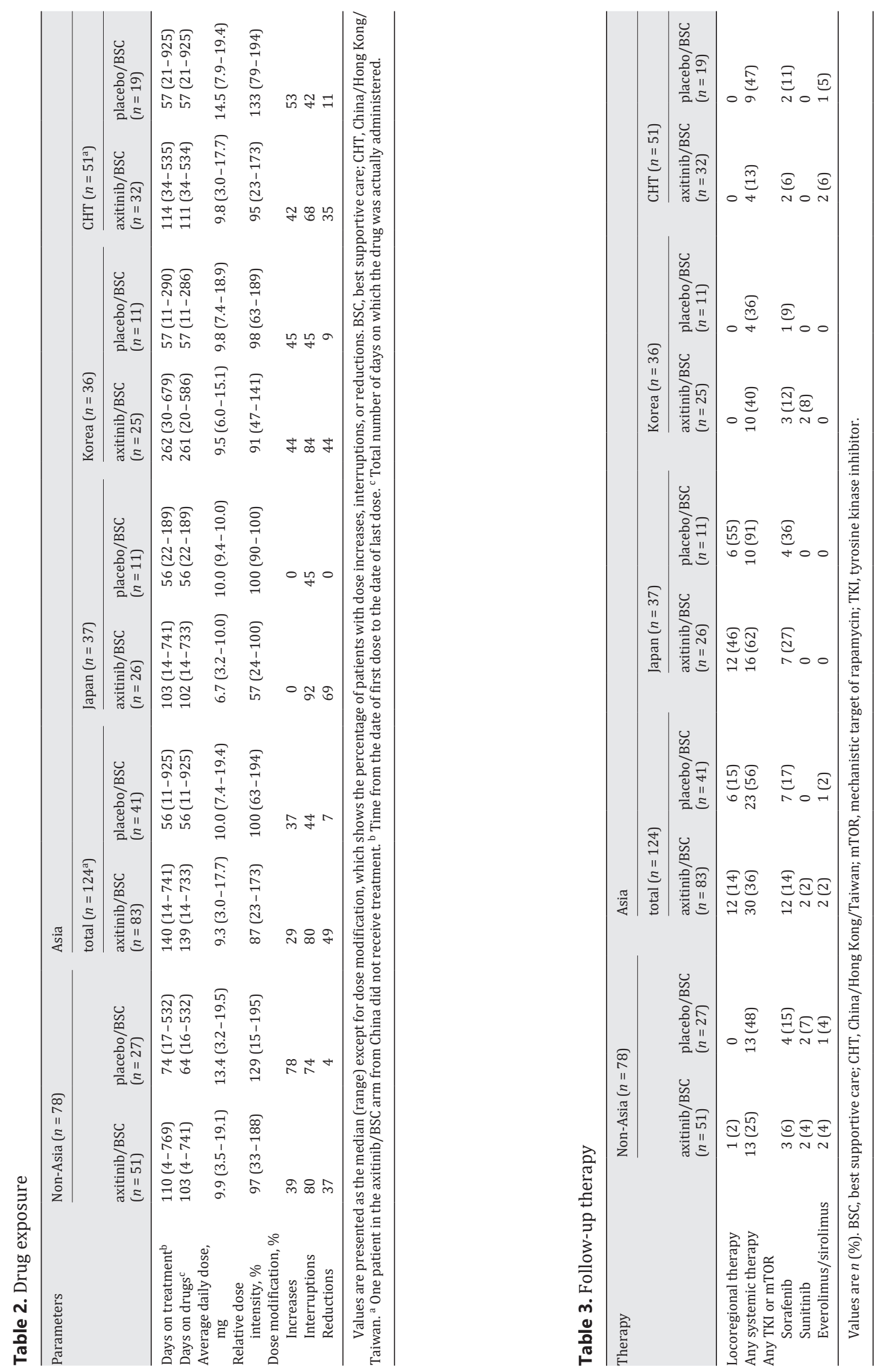
study treatment, mostly due to objective progression/relapse or AEs (online suppl. Material S1; for all online suppl. material, see www.karger.com/doi/10.1159/000484620).

Within each region, the patients in the axitinib/BSC arm were on treatment for a significantly longer duration than those in the placebo/BSC arm (Table 2). Thus, the median time on the drug (i.e., the total number of days on which the drug was actually administered) in the axitinib/BSC arm was longer than in the placebo/BSC arm, especially among the patients from Korea (261 vs. 57 days). However, more frequent dose interruptions or dose reductions were observed with axitinib/BSC than with placebo/BSC treatment within each region (Table 2). When comparing the different regions, Japan had a higher percentage of patients with axitinib dose reductions than non-Asia, Korea, or CHT ( 69 vs. 37 vs. 44 vs. $35 \%$ ), and none of the Japanese patients had an increase in axitinib dose. Consequently, the patients in Japan received a lower axitinib dose than the patients in non-Asia, Korea, or CHT: an average daily axitinib dose of $6.7 \mathrm{mg}$ in Japan compared with $9.9 \mathrm{mg}$ in non-Asia, $9.5 \mathrm{mg}$ in Korea, and $9.8 \mathrm{mg}$ in CHT. The lowest median axitinib relative dose intensity was reported among the patients in Japan (57\%), compared with the median relative dose intensities of $91-97 \%$ achieved among the patients in non-Asia, Korea, or CHT.

\section{Follow-Up Therapy}

Geographical differences were seen in the use of follow-up locoregional or systemic therapies (Table 3). The number of patients in the axitinib/BSC and the placebo/BSC arm, respectively, receiving post-study locoregional therapy was higher in Japan (46 and 55\%) than in Korea ( 0 and $0 \%)$, CHT ( 0 and $0 \%)$, or non-Asia ( 2 and $0 \%)$. Also, a higher percentage of patients (70\% of all patients) in Japan received follow-up systemic therapy, including hepatic arterial infusion chemotherapy, compared with those in Korea, CHT, or non-Asia (39, 25, and $33 \%$, respectively). Sorafenib was the most frequently used tyrosine kinase inhibitor in follow-up therapy in all regions.

\section{Efficacy}

In the subgroup analysis, there was no statistically significant difference in OS between the axitinib/BSC and the placebo/BSC arm observed among the patients from non-Asia, from Asia, or from the Asian subgroups (Fig. 1). Median OS in the axitinib/BSC and the placebo/ BSC arm, respectively, was 12.3 months (95\% CI 9.5-14.9) and 11.2 months (95\% CI 7.9-16.0) in non-Asia (HR 0.977, 95\% CI 0.572-1.670; $p=0.4647$ ) and 13.5 months (95\% CI 9.2-18.1) and 6.3 months (95\% CI 3.1-11.6) in Asia (HR 0.832, 95\% CI 0.539-1.283; $p=0.2018$ ). The HR between the two treatment arms was 0.836 (95\% CI 0.378-1.849; $p=0.3319)$ in Japan, 0.925 (95\% CI 0.359-2.382; $p=0.4358$ ) in Korea, and 0.787 (95\% CI 0.419-1.480; $p=0.2262$ ) in CHT.

In an exploratory analysis in which patients intolerant to prior antiangiogenic therapy were excluded from the data set, OS favored axitinib/BSC over placebo/BSC in Asia, especially in Japan (Fig. 2). In non-Asia, median OS in the axitinib/BSC and the placebo/BSC arm, respectively, was 12.3 months (95\% CI 9.5-14.9) and 9.8 months (95\% CI 6.8-13.3) (HR 0.700, 95\% CI 0.373-1.316; $p=0.1318)$, whereas in Asia it was 12.4 months (95\% CI 7.4-16.4) and 5.2 months (95\% CI 3.1-9.9) (HR 0.653, 95\% CI 0.415-1.027; $p=0.0312$ ). The HR between the axitinib/BSC and the placebo/BSC arm reached statistical significance among the patients from Japan $(0.251,95 \%$ CI $0.097-0.650 ; p=0.0011)$, but not among the patients from Korea $(0.780,95 \%$ CI $0.283-2.150 ; p=0.3150)$ or CHT $(0.918,95 \%$ CI $0.480-1.756 ; p=0.3954)$. In Japan, OS with axitinib/BSC was significantly ( $p=0.0011)$ longer than with placebo/BSC (median 13.6 months [95\% CI 5.0-19.7] vs. 4.7 months [95\% CI 2.8-9.2]).

PFS remained significantly longer in the axitinib/BSC arm than in the placebo/BSC arm in Asia (HR 0.556, 95\% CI 0.370-0.835; $p=0.0023$ ) but not in non-Asia (HR 0.745, 95\% CI 
Kudo et al.: Regional Differences in Efficacy, Safety, and Biomarkers for Second-Line

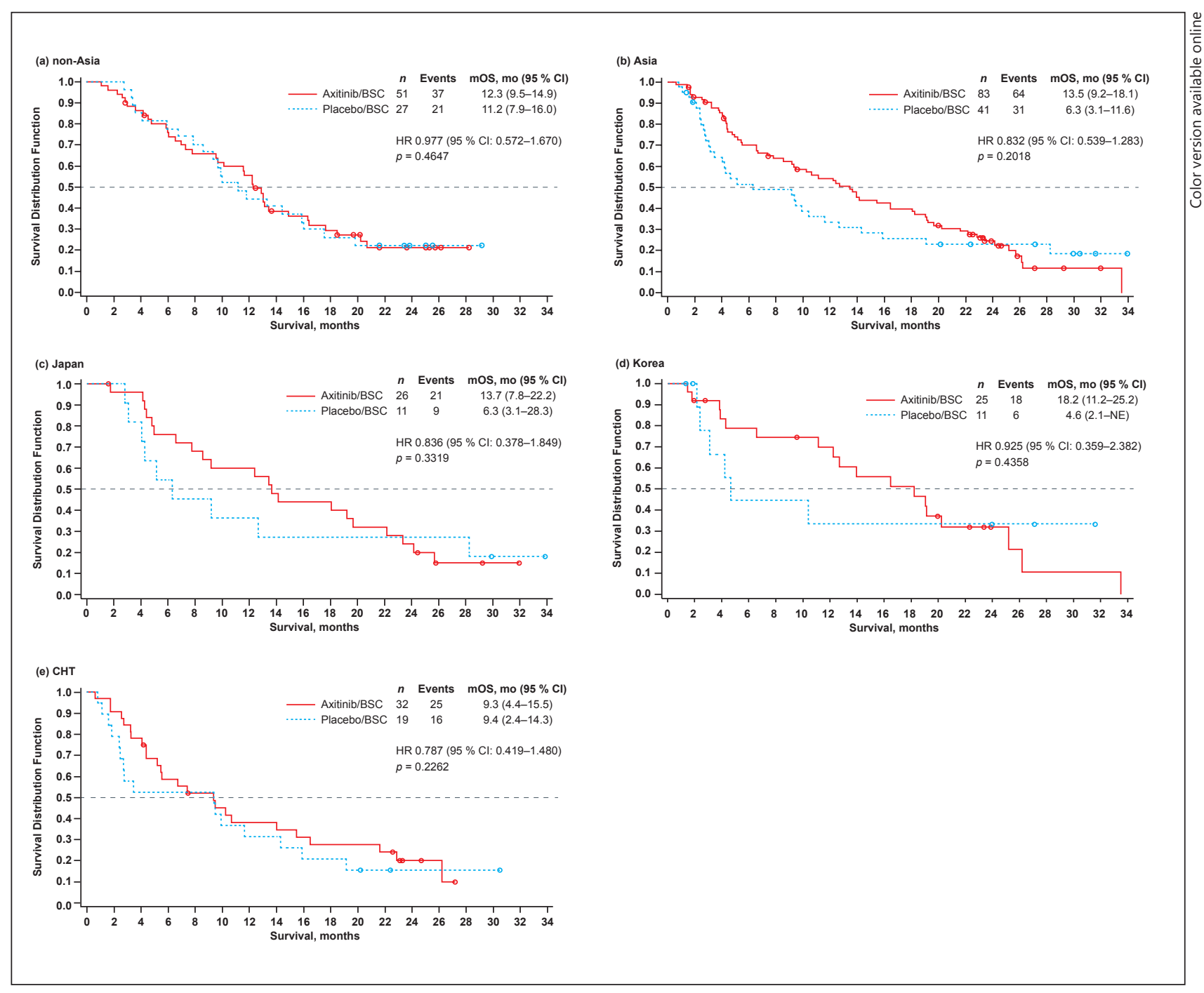

Fig. 1. Kaplan-Meier estimates for OS of patients from non-Asia (a), Asia (b), Japan (c), Korea (d), and CHT (e). $p$ values are based on an unstratified one-sided log-rank test. The primary data are available online. BSC, best supportive care; CHT, China/Hong Kong/Taiwan; CI, confidence interval; HR, hazard ratio; mo, months; mOS, median overall survival; NE, not estimable; OS, overall survival.

$0.416-1.335 ; p=0.1590$ ) (online suppl. Material S2). Median PFS among the axitinib/BSCtreated Asian patients was 3.6 months (95\% CI 1.9-4.6) as compared with 1.8 months (95\% CI 1.7-1.9) among the placebo/BSC-treated Asian patients, whereas among the non-Asian patients, median PFS was 3.7 months (95\% CI 1.9-8.8) with axitinib/BSC compared with 3.6 months (95\% CI 1.9-3.9) with placebo/BSC. Among the Asian subgroups, the difference in PFS favoring axitinib/BSC over placebo/BSC was statistically significant for Japan (HR 0.325 , 95\% CI 0.140-0.753; $p=0.0032$ ), but not for Korea (HR 0.613, 95\% CI 0.277-1.356; $p=$ 0.1046 ) or CHT (HR $0.715,95 \%$ CI $0.389-1.315 ; p=0.1460$ ) (online suppl. Material S2). Median PFS with axitinib/BSC and with placebo/BSC, respectively, was 3.6 months $(95 \% \mathrm{CI}$ 2.0-7.4) and 1.9 months (95\% CI 1.0-1.9) in Japan. 
Kudo et al.: Regional Differences in Efficacy, Safety, and Biomarkers for Second-Line

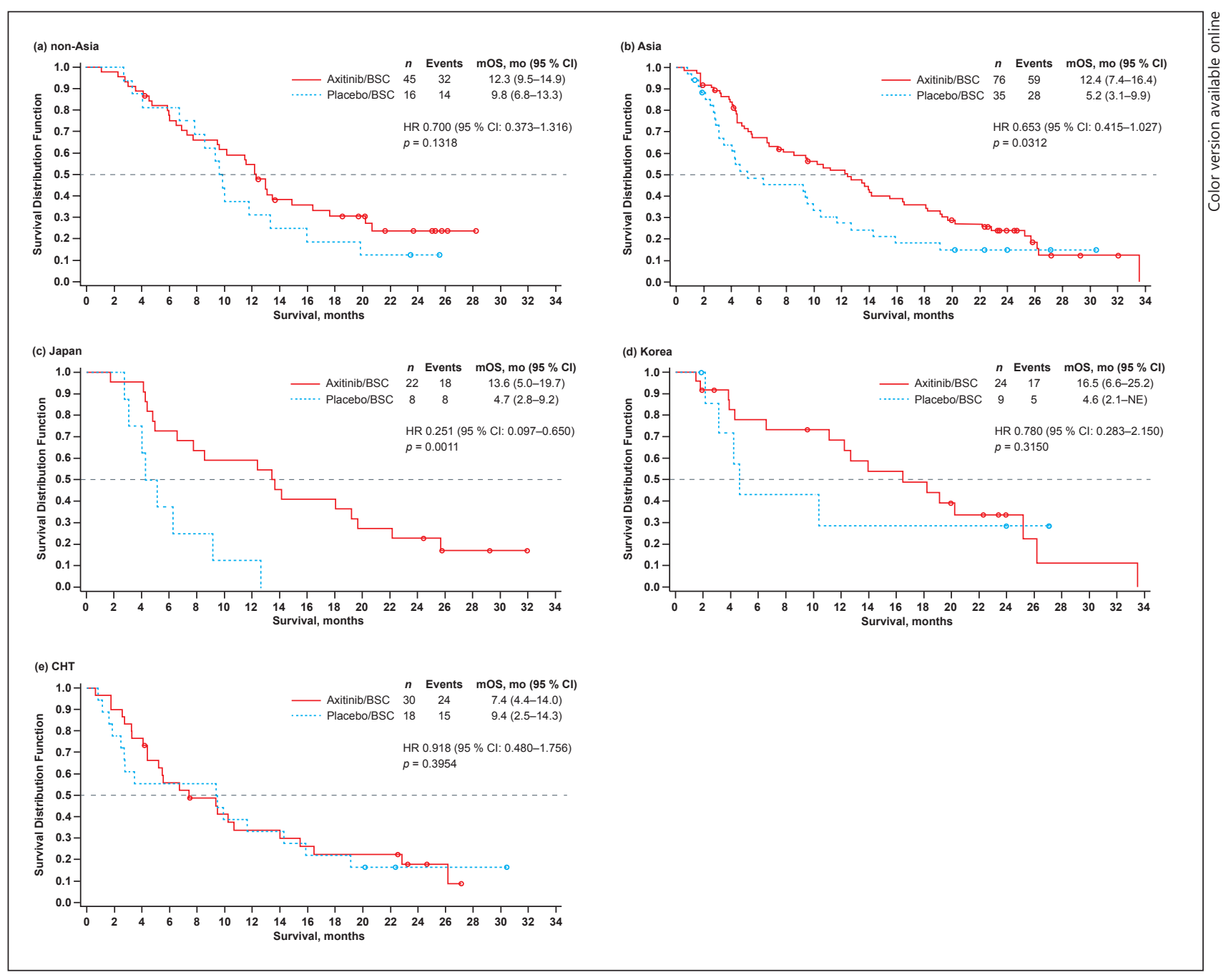

Fig. 2. Kaplan-Meier estimates for OS, excluding patients who were intolerant to prior antiangiogenic therapy, of patients from non-Asia (a), Asia (b), Japan (c), Korea (d), and CHT (e). $p$ values are based on an unstratified one-sided log-rank test. BSC, best supportive care; CHT, China/Hong Kong/Taiwan; CI, confidence interval; HR, hazard ratio; mo, months; mOS, median overall survival; NE, not estimable; OS, overall survival.

\section{Safety}

Overall, axitinib/BSC was well tolerated by both patients from non-Asia and those from Asia, although the incidence rates for several AEs were elevated compared with placebo/BSC (Table 4). For example, hypertension, diarrhea, and hypothyroidism were $>20 \%$ higher with axitinib/BSC than with placebo/BSC in both the non-Asian and the Asian patients. Decreased appetite, weight decrease, dysphonia, hand-foot syndrome, and proteinuria were $>20 \%$ higher with axitinib/BSC than with placebo/BSC only among the Asian patients, whereas asthenia (but not fatigue) and nausea were $>20 \%$ higher with axitinib/BSC than with placebo/ BSC only among the non-Asian patients.

Among the Asian subgroups, the incidence rates of several AEs varied substantially between the axitinib/BSC- and the placebo/BSC-treated patients, as well as between the Asian subgroups. The rate of hypertension was particularly higher (85\%) among the axitinib/ 
Kudo et al.: Regional Differences in Efficacy, Safety, and Biomarkers for Second-Line Axitinib in Patients with Advanced Hepatocellular Carcinoma

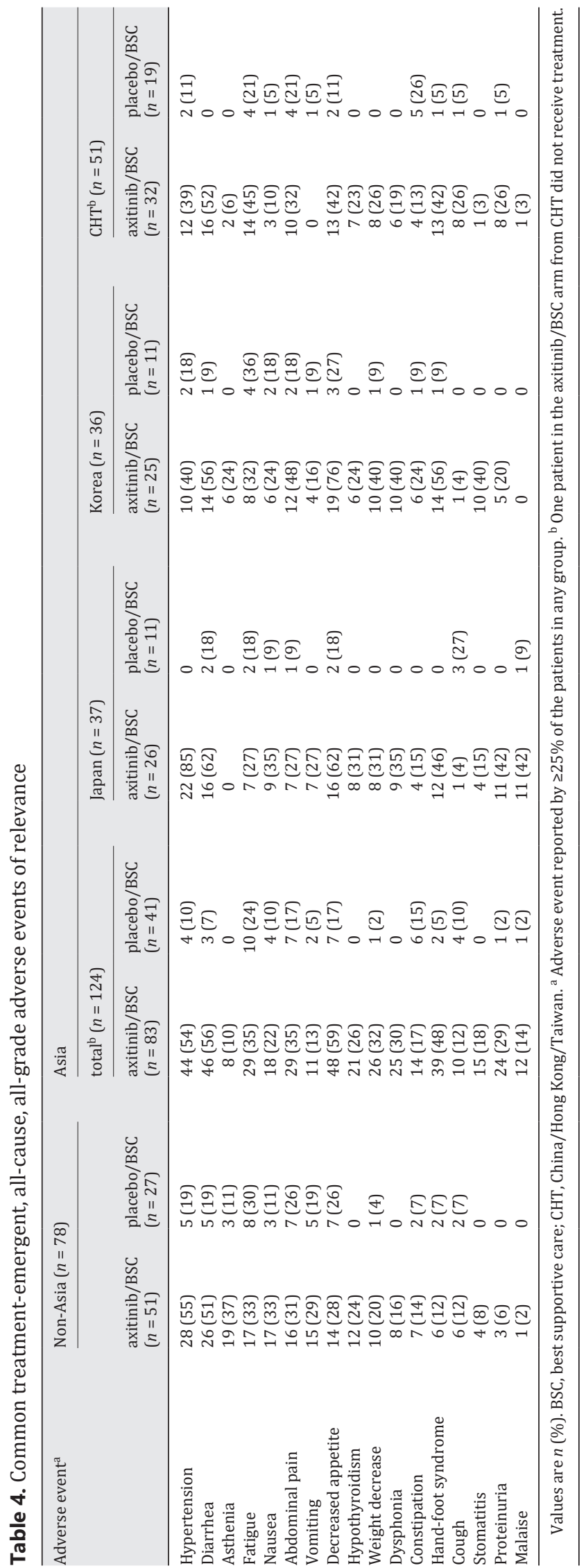




\section{Liver

Kudo et al.: Regional Differences in Efficacy, Safety, and Biomarkers for Second-Line

BSC-treated Japanese patients than among the placebo/BSC-treated Japanese patients (0\%) or the axitinib/BSC-treated patients from Korea (40\%) or CHT (39\%). Nausea and vomiting were also more frequently reported by the Japanese patients receiving axitinib/BSC compared with those receiving placebo/BSC or the patients from Korea or CHT who received axitinib/ BSC, but the reason for the higher incidence of nausea among the Japanese patients is unknown. Decreased appetite and stomatitis were more common in the axitinib/BSC-treated patients from Korea, whereas cough was more common in the axitinib/BSC-treated patients from CHT. Malaise had a higher incidence in the axitinib/BSC-treated Japanese patients than in the axitinib-treated patients from Korea or CHT (42 vs. 0 or $3 \%$ ). However, a plausible reason for this observation might be the different definition of malaise in these countries, since the incidence rate was lower in Japan than in Korea or CHT for AEs that could have been used interchangeably: asthenia $(0,24$, and $6 \%$, respectively) and fatigue $(27,32$, and $45 \%$, respectively).

All-cause grade $\geq 3$ AEs reported in $\geq 20 \%$ of the axitinib/BSC-treated patients in non-Asia versus Asia were hypertension (18 vs. $30 \%$ ), diarrhea ( 22 vs. $20 \%$ ), and hand-foot syndrome (4 vs. 22\%). Serious AEs associated with axitinib/BSC were reported in $51 \%$ of the patients from non-Asia versus 44\% of those from Asia; among the Asian subgroups, 46, 44, and 42\% of the patients treated with axitinib/BSC in Japan, Korea, and CHT, respectively, reported serious AEs.

The proportion of patients who had AEs requiring axitinib dose reductions was higher in Asia than in non-Asia (41 vs. 24\%), but a lower proportion of patients had AEs leading to treatment discontinuation in Asia than in non-Asia (22 vs. 39\%). Among the Asian subgroups, a higher percentage of patients had AEs requiring axitinib dose reductions in Korea than in Japan or CHT ( 60 vs. 42 or $26 \%$ ), but fewer patients had AEs leading to treatment discontinuations ( 8 vs. 23 or $32 \%$ ).

\section{Predictive and/or Prognostic Factors}

From the blood samples collected at baseline, RNA of sufficient quality for Agilent microarray analysis was generated from a majority of the patients $(84 \%, n=170$, including 72 nonAsians and 98 Asians). The predictive and/or prognostic potential of baseline levels of 174 circulating miRNAs for OS were evaluated, including those known to be associated with angiogenesis or tumor growth. The baseline characteristics of the patients included in the analyses were found to be balanced between the treatment arms (online suppl. Material S3).

The analysis identified several circulating miRNAs using the Cox proportional hazards model for OS. Positive (FDR $=0.2$ ) predictive effects associated with increased OS were observed for 3 miRNAs (hsa-miR-5684, hsa-miR-1224-5p, and hsa-miR-513c-5p) in the patients from Asia, but not in those from non-Asia. Another miRNA (hsa-miR-6075) was positively (FDR $=0.2$ ) predictive of survival in the patients from non-Asia, but not in those from Asia. A total of 4 circulating miRNAs were found to be statistically significantly (FDR $=0.2$ ) associated with longer OS (HR ranging from 0.08 to 0.33 ) when present versus absent in the overall, Asian, or non-Asian population (Fig. 3). In the Asian patients treated with axitinib/ BSC, median (95\% CI) OS was 19.1 months (9.2-25.2) if circulating hsa-miR-1224-5p was present, compared with 8.6 months (5.5-14.1) if absent. In the placebo/BSC arm, median (95\% CI) OS was 6.3 months (2.8-12.7) if present, and not estimable (1.8 to not estimable) if absent. Median OS in the presence versus absence of each of these 4 miRNAs is summarized in Table 5.

As reported elsewhere [7], the previous analysis based on the unstratified log-rank test unadjusted for multiple comparisons revealed an association between a low baseline serum level of E-selectin or SDF-1 and longer OS among all axitinib/BSC-treated patients compared with placebo/BSC-treated patients. Furthermore, in that report the Cox proportional hazards 
Kudo et al.: Regional Differences in Efficacy, Safety, and Biomarkers for Second-Line Axitinib in Patients with Advanced Hepatocellular Carcinoma

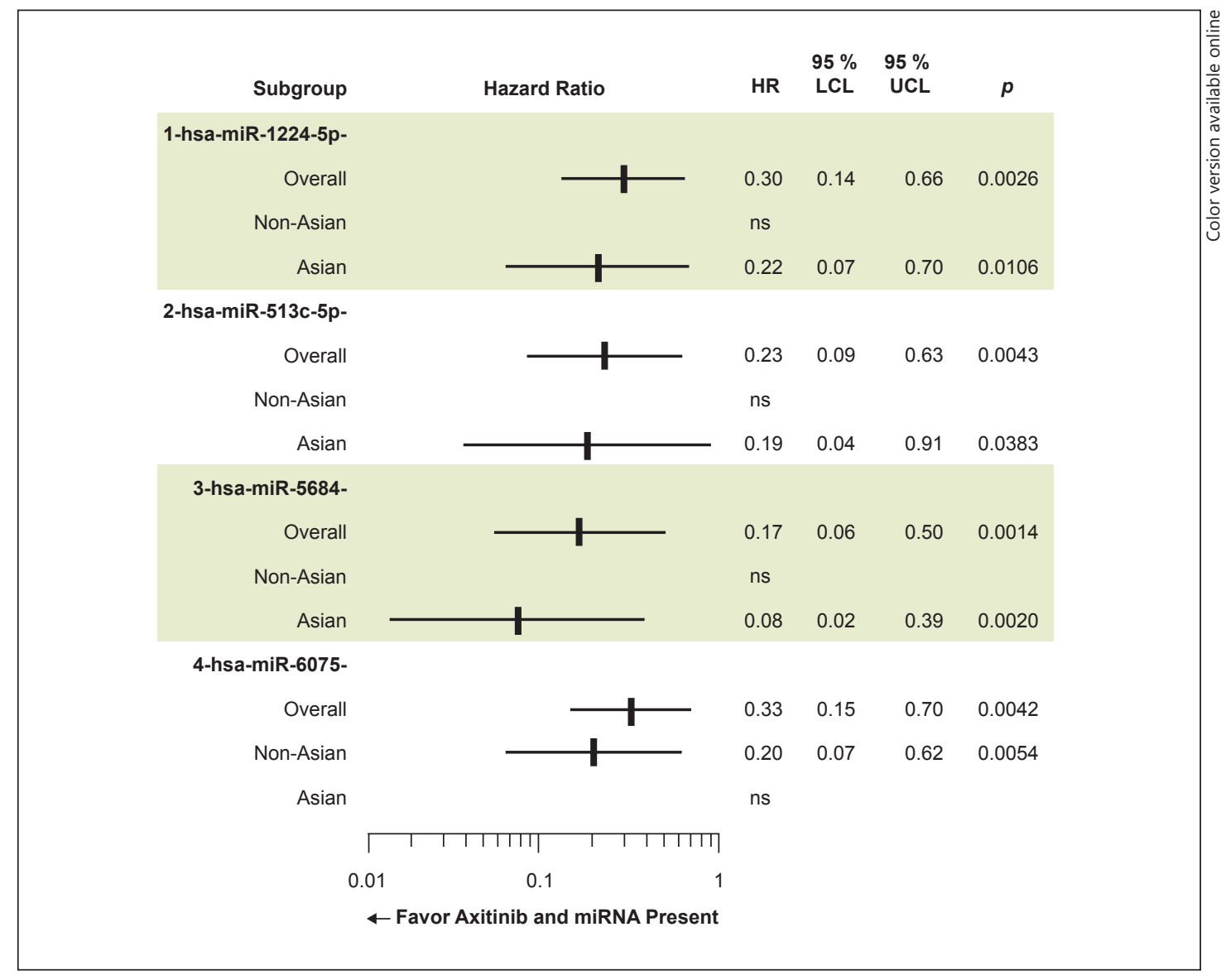

Fig. 3. Predictive circulating microRNA subgroup analysis of overall survival in the overall, non-Asian, and Asian population. HR, hazard ratio; LCL, lower confidence limit; miR, microRNA; ns, not significant; UCL, upper confidence limit.

model showed a lower-than-median baseline level of IL-6, E-selectin, IL-8, Ang-2, MIF, or soluble c-MET to be a potential positive prognostic factor for OS in HCC. In the current subgroup analyses, the significant association between a lower-than- or equal-to-median baseline serum level of SDF-1 ( $p_{\text {unadjusted }}=0.0026$ ) and longer OS was observed in the Asian patients who were treated with axitinib/BSC compared with those treated with placebo/BSC (FDR $=0.2)$. Among the non-Asian patients, no association was identified between any of the soluble proteins tested and OS.

\section{Discussion}

The current exploratory analyses of the efficacy and safety data from the phase II study of axitinib in advanced HCC were conducted in order to identify demographics and baseline characteristics of patients who might best benefit from axitinib treatment and to select more suitable target populations for future trials in advanced HCC. The analyses showed several interesting findings. First, some geographical differences in demographics and baseline characteristics were observed between patients with HCC enrolled in non-Asia and those enrolled in Asia, as well as between different Asian ethnicities. Notably, the percentage of patients with 
Kudo et al.: Regional Differences in Efficacy, Safety, and Biomarkers for Second-Line Axitinib in Patients with Advanced Hepatocellular Carcinoma

Table 5. Median OS stratified by select microRNAs in the axitinib/BSC and placebo/BSC arms

\begin{tabular}{|c|c|c|c|c|c|c|c|c|}
\hline \multirow[t]{3}{*}{$\mathrm{miR}$} & \multicolumn{8}{|c|}{ Median OS (95\% CI), months } \\
\hline & \multicolumn{4}{|c|}{ axitinib/BSC } & \multicolumn{4}{|c|}{ placebo/BSC } \\
\hline & $n$ & miR present & $n$ & miR absent & $n$ & miR present & $n$ & miR absent \\
\hline \multicolumn{9}{|c|}{ hsa-miR-1224-5p } \\
\hline Overall & 63 & $13.9(10.2-19.1)$ & 48 & $12.2(6.7-14.1)$ & 36 & $8.9(4.1-11.6)$ & 23 & $14.4(7.9-\mathrm{NE})$ \\
\hline Non-Asian & 31 & $12.3(6.9-17.6)$ & 17 & $13.0(4.6-20.2)$ & 15 & $9.3(3.4-13.3)$ & 11 & $14.4(7.9-\mathrm{NE})$ \\
\hline Asian & 32 & $19.1(9.2-25.2)$ & 31 & $8.6(5.5-14.1)$ & 21 & $6.3(2.8-12.7)$ & 12 & NE (1.8-NE) \\
\hline \multicolumn{9}{|c|}{ hsa-miR-513c-5p } \\
\hline Overall & 83 & $13.5(11.6-18.2)$ & 28 & $7.8(4.6-13.9)$ & 47 & $9.3(4.3-11.2)$ & 12 & $19.8(1.8-\mathrm{NE})$ \\
\hline Non-Asian & 39 & $12.3(9.7-13.5)$ & 9 & $14.9(2.8-20.7)$ & 20 & $9.5(4.1-13.3)$ & 6 & $18.7(11.8-\mathrm{NE})$ \\
\hline Asian & 44 & $18.2(8.6-23.4)$ & 19 & $7.8(3.9-13.7)$ & 27 & $9.2(3.1-12.7)$ & 6 & NE (1.1-NE) \\
\hline \multicolumn{9}{|l|}{ hsa-miR-5684 } \\
\hline Overall & 93 & $13.5(11.5-16.5)$ & 18 & $8.9(3.9-18.1)$ & 48 & $9.4(4.1-12.7)$ & 11 & NE (7.9-NE) \\
\hline Non-Asian & 42 & $12.9(9.5-14.9)$ & 6 & $15.4(3.0-20.7)$ & 21 & $10.0(5.9-15.9)$ & 5 & 11.8 (7.9-NE) \\
\hline Asian & 51 & $16.4(8.6-22.2)$ & 12 & $6.1(1.7-12.4)$ & 27 & $4.6(2.8-12.7)$ & 6 & NE (4.3-NE) \\
\hline \multicolumn{9}{|l|}{ hsa-miR-6075 } \\
\hline Overall & 62 & $16.4(10.2-22.2)$ & 49 & $12.2(6.7-13.7)$ & 35 & $9.2(4.2-12.7)$ & 24 & 11.8 (7.9-NE) \\
\hline Non-Asian & 28 & $12.9(7.3-\mathrm{NE})$ & 20 & $12.2(4.6-14.9)$ & 14 & $7.7(3.4-13.3)$ & 12 & $16.0(9.3-\mathrm{NE})$ \\
\hline Asian & 34 & $18.2(5.5-25.2)$ & 29 & $11.2(6.6-14.1)$ & 21 & $9.4(4.2-15.9)$ & 12 & $4.0(1.6-\mathrm{NE})$ \\
\hline
\end{tabular}

BSC, best supportive care; CI, confidence interval; miR, microRNA; NE, not estimable; OS, overall survival.

hepatitis B, one of the primary causes of HCC, was significantly higher in Asia (excluding Japan) than in non-Asia, especially in CHT, where 95\% of the patients had hepatitis B. Hepatitis $\mathrm{C}$, another potential cause of HCC, was prevalent among Japanese patients. The percentage of patients with baseline extrahepatic spreads with intrahepatic tumors and BCLC stage C, and thus a less favorable prognosis, was also higher among patients in Asia than among those in non-Asia. These results suggest a different etiology of HCC in patients from different regions and ethnicities, which would have confounded the interpretation of the results of the subgroup analyses. Additionally, despite the fact that the randomization of patients was stratified by tumor invasion (i.e., the presence or absence of extrahepatic spread and/or tumor vascular invasion) in the overall population, minor differences in baseline characteristics - such as extrahepatic spread with intrahepatic tumors, tumor vascular invasion, and hepatitis $\mathrm{C}$ were observed between the axitinib/BSC and the placebo/BSC treatment arm in the Asian subgroups, especially in Japan, further making the interpretation of the data more challenging.

Second, there was no statistically significant difference in OS between axitinib/BSCtreated and placebo/BSC-treated patients in non-Asia or in Asia. A lack of clinical benefit from adding axitinib to BSC was also evident in the Asian subgroups. However, in an exploratory analysis in which patients who were intolerant to prior antiangiogenic therapy were excluded, there was a difference in OS between the two treatment arms, favoring axitinib/BSC among Japanese patients. Finally, our analyses suggest that baseline levels of 4 circulating miRNAs, including hsa-miR-5684 and hsa-miR-1224-5p, as well as serum protein SDF-1 might have some predictive value for OS with axitinib and/or prognostic value in HCC patients, which warrants further investigation.

Apart from the geographical differences noted in baseline characteristics of the HCC patients, the analyses revealed an interesting disparity in clinical practice relative to treatment regimens between the Asian group, the non-Asian group, and the Asian subgroups. Patients in CHT had similar treatment schedules and dose modifications to non-Asian patients, 


\section{Liver

Kudo et al.: Regional Differences in Efficacy, Safety, and Biomarkers for Second-Line

Axitinib in Patients with Advanced Hepatocellular Carcinoma

whereas patients from Korea were on axitinib/BSC treatment more than twice as long as patients from other regions. Although Japanese and Korean patients had more axitinib dose reductions due to AEs than Chinese patients, they had fewer axitinib discontinuations due to AEs. Japanese patients had the most axitinib dose reductions and temporary dose interruptions due to any cause, and with none of the Japanese patients receiving dose increases, their axitinib relative dose intensity was the lowest. Based on these observations, it is tempting to postulate that more aggressive axitinib treatment modifications in Japan might have contributed to more favorable efficacy outcomes, including both PFS and OS, than those in Korea, CHT, or non-Asia. However, it is likely that other factors, such as more frequent use of post-study therapies, must also have accounted for the outcome. It is noteworthy that similar regional disparities in treatment practice patterns and patient baseline characteristics have been reported in a subgroup analysis of a randomized phase III trial of ramucirumab in advanced HCC, which the authors saw as some of the likely reasons for achieving survival benefits in Japanese patients $(n=93)$ [9] but not in the total population $(n=565)$ [4]. These findings observed in clinical trials seem to reflect the real-world setting, as seen in a subgroup analysis of a prospective, international, noninterventional study of sorafenib in patients with unresectable HCC [10].

In this analysis, median OS with axitinib/BSC in non-Asian patients was marginally shorter than in Asian patients, although non-Asian patients had better prognostic factors at baseline. Among Asian ethnicities, median OS was longest in axitinib/BSC-treated Korean patients and shortest in Chinese patients. Patients in Korea were on axitinib/BSC treatment for the longest period, and patients in Japan received the most locoregional or systemic follow-up therapies, which might explain, at least in part, the numerically longer median OS of these patients. Interestingly, median OS in the placebo/BSC arm was shorter in patients from Korea (4.6 months) and Japan (6.3 months) than in those from CHT (9.4 months) or non-Asia (11.2 months), especially in light of the fact that a higher percentage of placebo/ BSC-treated patients in Japan underwent post-study locoregional and/or systemic therapy. Incidentally, the treatment duration was substantially shorter in the placebo/BSC arm than in the axitinib/BSC arm in all the groups.

It was reported that patients who discontinued prior antiangiogenic therapy due to AEs had a better prognosis than those who discontinued treatment due to disease progression or liver failure [11]. In the current analysis, the placebo/BSC arm had a higher proportion of patients who were intolerant to prior antiangiogenic therapy than the axitinib/BSC arm, except for the CHT subgroup. This might explain, at least in part, the improvement in HR favoring axitinib/BSC observed in all Asian regions except CHT when patients intolerant to prior antiangiogenic therapy were excluded from the analysis. In particular, a potentially better OS favoring axitinib/BSC over placebo/BSC was seen in patients from Japan. It is possible that aggressive post-study treatment with sorafenib and/or locoregional therapy resulted in prolonged postprogression survival (PPS), especially in patients who had discontinued prior sorafenib due to AEs, since those patients were generally in good health. Postprogression survival, defined as the time between the last day of treatment and death or last visit recorded, has been demonstrated to strongly correlate with median OS $[11,12]$. Additionally, if there are more patients in the placebo/BSC arm who are intolerant to sorafenib but receive sorafenib rechallenge post-study treatment, those patients may benefit from sorafenib rechallenge after failure of study treatment, as has been reported in another study [13].

The nature of AEs reported by patients treated with axitinib/BSC was similar between non-Asia and Asia. However, some AEs, such as asthenia, were more frequent in non-Asia, whereas decreased appetite, hand-foot syndrome, and proteinuria were more common in Asia. Unexpectedly, the incidence rates for several AEs varied between different Asian ethnicities, which may be attributed not only to some minor genomic variations in drug-metabo- 


\section{Liver}

\begin{tabular}{l|l}
\hline \multicolumn{2}{|l}{ Liver Cancer 2018;7:148-164 } \\
\hline DOI: 10.1159/000484620 & $\begin{array}{l}\text { @ 2017 S. Karger AG, Basel } \\
\text { www.karger.com/lic }\end{array}$ \\
\hline
\end{tabular}

Kudo et al.: Regional Differences in Efficacy, Safety, and Biomarkers for Second-Line Axitinib in Patients with Advanced Hepatocellular Carcinoma

lizing enzymes and/or transporters $[14,15]$ but also to differences in clinical practice in monitoring and management of common AEs. It should be mentioned that although axitinib population pharmacokinetic analysis for RCC has indicated that Japanese ethnicity is associated with decreased systemic clearance, the magnitude of the effect was considered such that dose adjustments on the basis of Japanese ethnicity were deemed unnecessary $[16,17]$.

There has been a growing list of targeted therapies that failed to show improved OS in sorafenib-refractory patients with advanced HCC in multinational, double-blind, randomized phase III trials. For instance, median OS for a selective dual inhibitor of VEGFR and the fibroblast growth factor receptor brivanib in combination with BSC was 9.4 months compared with 8.2 months for placebo/BSC (HR 0.89; $p=0.3307$ ), indicating a lack of survival benefit for brivanib in HCC [3]. Targeting VEGFR using the monoclonal antibody ramucirumab did not lead to prolongation of survival compared with placebo in the overall population (HR $0.87 ; p=0.14$ ) [4], despite findings from subgroup analyses that ramucirumab improved OS and PFS among Japanese patients [9]. Another targeted agent, everolimus - an inhibitor of mechanistic target of rapamycin - failed to improve OS over placebo (HR 1.05; $p=0.68$ ) when added to BSC in patients with advanced HCC previously treated with sorafenib [5]. However, recently, a phase III trial of another multitargeted tyrosine kinase inhibitor, regorafenib (ClinicalTrials.gov No. NCT01774344, RESORCE trial), met its primary endpoint of a statistically significant improvement in OS in sorafenib-refractory HCC [18]. In that study, only patients who progressed on sorafenib were enrolled, excluding sorafenib-intolerant patients.

The current study has some limitations, the most critical one being that it was an exploratory analysis, and although the patient baseline characteristics were generally comparable between the two treatment arms, there were some minor differences, including tumor invasion, in the Asian group and the Asian subgroups, which could have impacted the results. There were also differences in the frequency of follow-up therapy, with the highest in Japan, which could have contributed to the outcomes. Another limitation is that confounding factors such as the level of hepatic functionality were not evaluated in the current study, and thus were not taken into account in the analyses. It should also be acknowledged that the sample size for some Asian subgroups (e.g., 11 patients each in the placebo/BSC arms in Japan and Korea) was too small to draw definite conclusions.

As in other similar studies, the lack of validated biomarkers that would predict treatment outcomes in metastatic HCC has prevented the selection of patients who might preferentially achieve better clinical outcomes with axitinib/BSC treatment. This study identified a set of circulating miRNAs (e.g., hsa-miR-5684 and hsa-miR-1224-5p) that may have the potential to serve as predictors of response to axitinib treatment in Asian patients with metastatic HCC. However, target genes for these miRNAs are currently unknown, and because the results are extremely preliminary with a potential imbalance in background characteristics - such as regarding tumor vascular invasion and/or extrahepatic spread - between subgroups consisting of small numbers of patients included in the biomarker analysis, additional investigations are necessary in a large group of patients to validate these results.

The current analyses further provided evidence that a low baseline level of SDF-1 was associated with longer OS in Asian patients receiving axitinib/BSC compared with placebo/BSC, as was observed in the overall population [7]. But this association was not observed in non-Asian patients. Since the pathophysiology of HCC and responses to blocking the VEGF/VEGFR signaling pathway are far more complex, additional studies are required to elucidate why these associations were preferentially observed in Asian patients but not in non-Asian patients.

In conclusion, addition of axitinib to BSC did not prolong survival over placebo/BSC in patients from non-Asia, Asia, or Asian subgroups. However, axitinib/BSC showed favorable OS versus placebo/BSC in patients from Asia, especially Japan, when patients intolerant to prior antiangiogenic therapy were excluded from the data set. The data suggest that patients 
who progress on prior systemic therapy might constitute a more suitable population for clinical trials of new agents in advanced HCC. Furthermore, PFS in the axitinib/BSC arm remained significantly longer than in the placebo/BSC arm in Asia, but not in non-Asia. Differences in demographics and baseline characteristics observed in patients from different geographical regions could potentially explain the more favorable OS seen in patients from regions such as Japan. In addition, appropriate axitinib dose modifications may play an important role in maintaining a longer duration of treatment, which could lead to better efficacy outcomes. The potential predictive and/or prognostic value of a set of 4 baseline miRNAs (including hsa-miR-5684 and hsa-miR-1224-5p) and soluble protein SDF-1 identified in this study warrants further investigation in a prospective study.

\section{Acknowledgments}

Medical writing support was provided by Mariko Nagashima, PhD, of Engage Scientific Solutions (Southport, CT, USA) and was funded by Pfizer.

\section{Statement of Ethics}

The study was conducted in accordance with the Declaration of Helsinki and the International Conference on Harmonization guidelines on Good Clinical Practice. The protocol was approved by an institutional review board or independent ethics committee at each center, and all patients provided written informed consent.

\section{Disclosure Statement}

M.K. has served as an adviser for Kowa, MSD, Bristol-Myers Squibb, Bayer, Chugai, and Taiho, has received honoraria from Bayer, Eisai, MSD, and Ajinomoto, and has received research funding from Chugai, Otsuka, Takeda, Taiho, Sumitomo Dainippon, Daiichi Sankyo, MSD, Eisai, Bayer, and AbbVie. Y.-K.K. has received a research grant from Bayer and Sanofi. E.A. has served as a consultant for Sanofi, IPSEN, Novartis, and Bayer, and has received travel/accommodations/expenses from Celgene. Y.U. and Y.F. are employed by Pfizer Japan, and Y.U. owns stock in Pfizer. M.J.L., O.V., and J.-F.M. are employed by and own stock in Pfizer. J.A.W. was employed by Pfizer at the time of the study; he is currently employed by Genentech/Roche. All the other authors declare that they have no conflict of interest.

\section{Funding Source}

This study was sponsored by Pfizer.

\section{References}

1 Ferlay J, Soerjomataram I, Ervik M, Dikshit R, Eser S, Mathers C, Rebelo M, Parkin DM, Forman D, Bray F: GLOBOCAN 2012 v1.0, Cancer Incidence and Mortality Worldwide: IARC CancerBase No. 11. Lyon, International Agency for Research on Cancer. http://globocan.iarc.fr (accessed May 18, 2016).

2 National Comprehensive Cancer Network: NCCN Clinical Practice Guidelines in Oncology (NCCN Guidelines) Hepatobiliary Cancers version 1. 2016. https://www.nccn.org/professionals/physician_gls/pdf/hepatobiliary.pdf (accessed May 19, 2016).

3 Llovet JM, Decaens T, Raoul JL, Boucher E, Kudo M, Chang C, Kang YK, Assenat E, Lim HY, Boige V, Mathurin P, Fartoux L, Lin DY, Bruix J, Poon RT, Sherman M, Blanc JF, Finn RS, Tak WY, Chao Y, Ezzeddine R, Liu D, Walters I, Park JW: Brivanib in patients with advanced hepatocellular carcinoma who were intolerant to sorafenib or for whom sorafenib failed: results from the randomized phase III BRISK-PS study. J Clin Oncol 2013;31:35093516. 
Kudo et al.: Regional Differences in Efficacy, Safety, and Biomarkers for Second-Line Axitinib in Patients with Advanced Hepatocellular Carcinoma

4 Zhu AX, Park JO, Ryoo BY, Yen CJ, Poon R, Pastorelli D, Blanc JF, Chung HC, Baron AD, Pfiffer TE, Okusaka T, Kubackova K, Trojan J, Sastre J, Chau I, Chang SC, Abada PB, Yang L, Schwartz JD, Kudo M: Ramucirumab versus placebo as second-line treatment in patients with advanced hepatocellular carcinoma following first-line therapy with sorafenib (REACH): a randomised, double-blind, multicentre, phase 3 trial. Lancet Oncol 2015; 16:859-870.

5 Zhu AX, Kudo M, Assenat E, Cattan S, Kang YK, Lim HY, Poon RT, Blanc JF, Vogel A, Chen CL, Dorval E, PeckRadosavljevic M, Santoro A, Daniele B, Furuse J, Jappe A, Perraud K, Anak O, Sellami DB, Chen LT: Effect of everolimus on survival in advanced hepatocellular carcinoma after failure of sorafenib: the EVOLVE-1 randomized clinical trial. JAMA 2014;312:57-67.

6 INLYTA $^{\circledR}$ (axitinib) prescribing information. Pfizer, 2012. http://labeling.pfizer.com/ShowLabeling. aspx?id=759.

7 Kang YK, Yau T, Park JW, Lim HY, Lee TY, Obi S, Chan SL, Qin S, Kim RD, Casey M, Chen C, Bhattacharyya H, Williams JA, Valota O, Chakrabarti D, Kudo M: Randomized phase II study of axitinib versus placebo plus best supportive care in second-line treatment of advanced hepatocellular carcinoma. Ann Oncol 2015;26:24572463.

8 Benjamini Y, Hochberg Y: Controlling the false discovery rate: a practical and powerful approach to multiple testing. JR Stat Soc Series B Methodol 1995;57:289-300.

9 Kudo M, Hatano E, Ohkawa S, Fujii H, Masumoto A, Furuse J, Wada Y, Ishii H, Obi S, Kaneko S, Kawazoe S, Yokosuka O, Ikeda M, Ukai K, Morita S, Tsuji A, Kudo T, Shimada M, Osaki Y, Tateishi R, Sugiyama G, Abada PB, Yang L, Okusaka T, Zhu AX: Ramucirumab as second-line treatment in patients with advanced hepatocellular carcinoma: Japanese subgroup analysis of the REACH trial. J Gastroenterol 2017;52:494-503.

10 Kudo M, Lencioni R, Marrero JA, Venook AP, Bronowicki JP, Chen XP, Dagher L, Furuse J, Geschwind JF, Ladrón de Guevara L, Papandreou C, Sanyal AJ, Takayama T, Yoon SK, Nakajima K, Lehr R, Heldner S, Ye SL: Regional differences in sorafenib-treated patients with hepatocellular carcinoma: GIDEON observational study. Liver Int 2016;36:1196-1205.

11 Iavarone M, Cabibbo G, Biolato M, Della Corte C, Maida M, Barbara M, Basso M, Vavassori S, Craxi A, Grieco A, Cammà C, Colombo M: Predictors of survival in patients with advanced hepatocellular carcinoma who permanently discontinued sorafenib. Hepatology 2015;62:784-791.

12 Terashima T, Yamashita T, Takata N, Nakagawa H, Toyama T, Arai K, Kitamura K, Yamashita T, Sakai Y, Mizukoshi E, Honda M, Kaneko S: Post-progression survival and progression-free survival in patients with advanced hepatocellular carcinoma treated by sorafenib. Hepatol Res 2016;46:650-656.

13 Nozawa M, Yamamoto Y, Minami T, Shimizu N, Hatanaka Y, Tsuji H, Uemura H: Sorafenib rechallenge in patients with metastatic renal cell carcinoma. BJU Int 2012;110(pt B):E228-E234.

14 Kurose K, Sugiyama E, Saito Y: Population differences in major functional polymorphisms of pharmacokinetics/pharmacodynamics-related genes in Eastern Asians and Europeans: implications in the clinical trials for novel drug development. Drug Metab Pharmacokinet 2012;27:9-54.

15 Liu JY, Qu K, Sferruzza AD, Bender RA: Distribution of the UGT1A1*28 polymorphism in Caucasian and Asian populations in the US: a genomic analysis of 138 healthy individuals. Anticancer Drugs 2007;18:693-696.

16 Rini BI, Garrett M, Poland B, Dutcher JP, Rixe O, Wilding G, Stadler WM, Pithavala YK, Kim S, Tarazi J, Motzer RJ: Axitinib in metastatic renal cell carcinoma: results of a pharmacokinetic and pharmacodynamic analysis. J Clin Pharmacol 2013;53:491-504.

17 Chen Y, Suzuki A, Tortorici MA, Garrett M, LaBadie RR, Umeyama Y, Pithavala YK: Axitinib plasma pharmacokinetics and ethnic differences. Invest New Drugs 2015;33:521-532.

18 Bruix J, Qin S, Merle P, Granito A, Huang Y-H, Bodoky G, Pracht M, Yokosuka O, Rosmorduc O, Breder V, Gerolami R, Masi G, Ross PJ, Song T, Bronowicki JP, Ollivier-Hourmand I, Kudo M, Cheng AL, Llovet JM, Finn RS, LeBerre MA, Baumhauer A, Meinhardt G, Han G; RESORCE Investigators: Regorafenib for patients with hepatocellular carcinoma who progressed on sorafenib treatment (RESORCE): a randomised, double-blind, placebo-controlled, phase 3 trial. Lancet 2017;389:56-66. 